Cinémas

Revue d'études cinématographiques

Journal of Film Studies

\title{
Pages liminaires
}

Volume 29, numéro 1, automne 2018

URI : https://id.erudit.org/iderudit/1071094ar

DOI : https://doi.org/10.7202/1071094ar

Aller au sommaire du numéro

Éditeur(s)

Cinémas

ISSN

1181-6945 (imprimé)

1705-6500 (numérique)

Découvrir la revue

Citer ce document

(2018). Pages liminaires. Cinémas, 29(1), 3-4. https://doi.org/10.7202/1071094ar

Ce document est protégé par la loi sur le droit d'auteur. L'utilisation des services d'Érudit (y compris la reproduction) est assujettie à sa politique d'utilisation que vous pouvez consulter en ligne.

https://apropos.erudit.org/fr/usagers/politique-dutilisation/
Cet article est diffusé et préservé par Érudit.

Érudit est un consortium interuniversitaire sans but lucratif composé de l'Université de Montréal, l'Université Laval et l'Université du Québec à Montréal. Il a pour mission la promotion et la valorisation de la recherche. https://www.erudit.org/fr/ 


\section{CiNémas}

Cinémas est une revue spécialisée consacrée d'abord aux études cinématographiques et aux travaux théoriques ou analytiques propres à stimuler une réflexion issue de la rencontre de différentes approches.

Accordant une attention particulière aux recherches sur les mutations en cours, au sein tant des pratiques créatrices que des discours théoriques, Cinémas est à l'écoute des nouveaux courants de pensée qui émergent du champ des études cinématographiques et milite en faveur d'un éclatement des disciplines; d'où son ouverture aux réflexions interdisciplinaires, aux nouveaux objets et aux nouvelles méthodes.

\section{Directeur}

Richard Bégin, Université de Montréal

\section{Éditrice}

Marnie Mariscalchi

\section{Comité éditorial}

Richard Bégin, Université de Montréal • Marta Boni, Université de Montréal • Philippe Mather, Campion College at the University of Regina • Viva Paci, Université du Québec à Montréal • Joëlle Rouleau, Université de Montréal • Aboubakar Sanogo, Carleton University

\section{Comité international}

Emmanuelle André, Université Paris 7 • Vincent Bouchard, Indiana University • Laurent Guido, Université Lille $3 \bullet$ Todd McGowan, University of Vermont • Dominique Nasta, Université libre de Bruxelles • Beate Ochsner, Universität Konstanz • Heui-Tae Park, Sungkyunkwan University • Leonardo Quaresima, Università di Udine • Maxime Scheinfeigel, Université Montpellier 3 • Adolfo Vera, Universidad de Valparaíso

\section{Évaluateurs}

Olivier Aïm • Bernard Andrieu • Muriel Andrin • Riccardo Antoniani • Sémir Badir • Mieke Bal • Simona Bealcovschi • Julie Beaulieu • Christa Blümlinger • Stéphany Boisvert - François Bovier • Évelyne Broudoux • Mathieu Capel • Silvio Celli • Tom Conley • JeanMichel Durafour • Thomas Elsaesser • Jean-Pierre Esquenazi • Giuseppe Fidotta • Marc Furstenau • Ronald Geerts • Omar Hachemi • Anne-Violaine Houcke • Matthieu Kolatte • Natacha Laurent • Mathias Lavin • Véronique Leduc • Thierry Lefebvre • Jean-Marc Leveratto - Emmanuel Licha • Philippe Ortel • Silvia Paggi • Chiara Palermo • Christian Poirier • Katalin Por • Michael Punt • Jacopo Rasmi • Julie Ravary-Pilon • Richard Rushton • Lori Saint-Martin • Valérie Schafer • Alexandre Sumpf • Pascale Thibaudeau • Benjamin Thomas - Myriam Tsikounas • Benoît Turquety • Haidee Wasson 
Cinémas est une revue indépendante gérée par un organisme sans but lucratif.

Elle reçoit le soutien du Conseil de recherches en sciences humaines du Canada et du Fonds de recherche du Québec - Société et culture, ainsi que l'appui de l'Université de Montréal (Département d'histoire de l'art et d'études cinématographiques, Faculté des arts et des sciences).

Elle est membre de l'Association canadienne des revues savantes et de la Société pour le développement des périodiques culturels québécois.

\section{Coordonnées}

Revue Cinémas

Université de Montréal,

Pavillon Lionel-Groulx

Case postale 6128, succursale Centre-ville

Montréal (Québec) H3C 3J7

Canada

5143436111 poste 3684

revue.cinemas@gmail.com

revue-cinemas.info

\section{Siège social}

Revue Cinémas

Université de Montréal,

Pavillon Lionel-Groulx

3150 rue Jean-Brillant, $2^{e}$ étage, local C2168

Montréal (Québec) H3T 1N8

Canada

\section{Dépôt légal}

Bibliothèque et Archives nationales

du Québec

Bibliothèque et Archives Canada

ISSN imprimé 1181-6945

ISSN numérique 1705-6500

ISBN imprimé 978-2-924826-08-9

ISBN PDF 978-2-924826-09-6

Ce numéro a été composé en Capitolium 2 (Gerard Unger, TypeTogether)

C Revue Cinémas, 2020. Tous droits réservés.

\author{
Distribution \\ Sodep \\ sodep.qc.ca \\ info@sodep.qc.ca
}

\section{Diffusion numérique}

Érudit

cine.erudit.org

client@erudit.org

Révision (textes français)

Noémie Thibodeau

Révision et traduction (textes anglais)

Timothy Barnard

\section{Correction d'épreuves (textes anglais)}

Jane Jackel

Mise en page

Édiscript

Couverture

SoManyGerrys

\section{Illustration de la couverture}

Scott Hernandez, CC BY-NC-ND 2.0

\section{Erratum}

Cinémas 28 (1) - Une erreur s'est glissée dans l'article de Nicolas Appelt intitulé "Usages des références historiques dans le cinéma syrien d'après 2011 \%. À la page 41, il faut lire: «En outre, le collectif tait la trajectoire du film de Fuller, transformé, dans la version abrégée et remontée du film de Weiss (Falkenau, Samuel Fuller témoigne, 2004), en un "récit fullerien" [...].»

espace pour le logo FSC

Imprimé sur des matériaux issus de forêts bien gérées certifiées FSC® et de matériaux recyclés. 